\title{
Implantação do programa hospital amigo do idoso: relato de experiência
}

\author{
Program implementation hospital friend of the elderly: \\ experience report
}

\author{
Geisiane de Souza Santos' ${ }^{1}$ Cristina Lavoyer Escudeiro² - Wbiratan de Lima Souza ${ }^{3}$ \\ Lucimere Maria dos Santos ${ }^{4}$ • Valquíria Carvalho Silva ${ }^{5}$ Fátima Helena do Espírito Santo ${ }^{6}$
}

\begin{abstract}
RESUMO
O envelhecimento populacional é um fenômeno mundial que trouxe grandes desafios para a sociedade, principalmente no setor saúde, que precisa se adequar às necessidades e demandas dos indivíduos idosos, que apresentam maior vulnerabilidade para agravos a saúde, incidência de hospitalizações e maiores custos nos serviços. Objetiva-se relatar um processo de implantação do programa Hospital Amigo do Idoso. Trata-se de um estudo descritivo, do tipo relato de experiência, acerca da implantação do programa Hospital Amigo do Idoso em um hospital da Baixada Litorânea do estado do Rio de Janeiro, descrevendo as mudanças ambientais e assistenciais ocorridas nesse processo. As mudanças estruturais e assistenciais na instituição, bem como as adequações dos serviços do hospital, levaram à uma assistência mais qualificada a essa clientela, desde o acolhimento até a alta hospitalar, com diminuição do tempo de internação e das complicações associadas a internação, redução dos custos com a hospitalização, bem como melhoria crescente dos indicadores de qualidade da assistência aos clientes idosos. Concluí-se que a implantação do programa Hospital Amigo do Idoso representou um esforço institucional que gerou mudanças fundamentais na instituiçãocom foco nas demandas inerentes ao envelhecimento.
\end{abstract}

Palavras-chave: Idoso; Hospitalização; Acolhimento.

\begin{abstract}
Population aging is a global phenomenon that has brought great challenges to society, especially in the health sector, which needs to fit the needs and demands of older people who are more vulnerable to health diseases, incidence of hospitalization and higher costs in services. The aim is to report a deployment process of the Hospital Friend of the Elderly program. This is a descriptive study, the type experience report concerning the implementation of the Baby Friendly Hospital for the Elderly program in a hospital in the Coast al Lowl and sof the state of Rio de Janeiro, describing the environmental and welfare changes in the process. The structural and welfare changes in the institution as well as the adequacy of hospital services, led to a more qualified assistance to this population, from the reception to the hospital with decreased hospital stay and complications associated with hospitalization, reducing the cost of hospitalization and increasing improvement of quality indicators of care for elderly clients. It concludes that the implementation of the Senior Friendly Hospital program represented an institutional effort that generated fundamental changes in the institution focused on the aged population demands.
\end{abstract}

Keywords: Aged; Hospitalization; Userembracement.

${ }^{1}$ Enfermeira. Especialista em Enfermagem do Trabalho. Mestranda do Programa de Pós-Graduação - Mestrado Profissional Enfermagem Assistencial da Escola de Enfermagem Aurora de Afonso Costa, da Universidade Federal Fluminense. Niterói. Rio de Janeiro - Brasil. E-mail: geisisantos10@hotmail.com

2 Enfermeira. Doutora em Enfermagem. Professora adjunta do departamento de enfermagem Médico-Cirúrgica da Escola de Enfermagem Aurora de Afonso Costa, da Universidade Federal Fluminense. Niterói. Rio de Janeiro - Brasil. E-mail: cristinalescudeiro@gmail.com

${ }^{3}$ Enfermeiro. Especialista em Enfermagem em Emergência Geral e Obstétrica. Mestrando do Programa de Pós-Graduação - Mestrado Profissional Enfermagem Assistencial da Escola de Enfermagem Aurora de Afonso Costa, da Universidade Federal Fluminense. Niterói. Rio de Janeiro - Brasil. E-mail: wbiratansouza@yahoo.com.br

${ }^{4}$ Enfermeira. Especialista em Enfermagem Oncológica. Mestranda do Programa de Pós-Graduação - Mestrado Profissional Enfermagem Assistencial da Escola de Enfermagem Aurora de Afonso Costa, da Universidade Federal Fluminense. Niterói. Rio de Janeiro - Brasil. E-mail: lucimere_santos@hotmail.com

${ }^{5}$ Enfermeira. Especialista em Saúde do Idoso. Mestranda do Programa de Pós-Graduação - Mestrado Profissional Enfermagem Assistencial da Escola de Enfermagem Aurora de Afonso Costa, da Universidade Federal Fluminense. Niterói. Rio de Janeiro - Brasil. E-mail: valquiria_carvalhosilva@hotmail.com

${ }^{6}$ Enfermeira. Doutora em Enfermagem. Professora adjunta do departamento de enfermagem Médico-Cirúrgica da Escola de Enfermagem Aurora de Afonso Costa, da Universidade Federal Fluminense. Coordenadora do Curso de Especialização em Enfermagem Gerontológica. Niterói. Rio de Janeiro - Brasil. E-mail: fatahelen@hotmail.com 


\section{INTRODUÇÃO}

O mundo está envelhecendo e, no Brasil, a radical transformação do perfil demográfico corresponde a uma das mais importantes modificações estruturais verificadas na sociedade, com redução das taxas de crescimento populacional e modificações na estrutura etária, decorrente da redução do número de crianças e adolescentes, concomitante ao crescimento da população em idade ativa e de pessoas idosas ${ }^{1}$.

De acordo com as projeções do Instituto Brasileiro de Geografia e Estatística, considerando o grupo etário de 60 anos ou mais, faixa etária definida como idosa segundo oEstatuto do Idoso, Lei $n^{\circ} 10.741$ de $1^{\circ}$ de outubro de 2003, ocorreu duplicação, no período de 2000 a 2020, ao passar de 13,9 para 28,3 milhões, elevando-se, em 2050, para 64 milhões. Mantidas as projeções de queda dos níveis da fecundidade no país, ter-se-á, em 2050, 226 idosos de 60 anos ou mais para cada 100 crianças e adolescentes ${ }^{1-2}$.

Além da transição demográfica, o Brasil tem experimentado uma transição epidemiológica, pois o perfil epidemiológico dos idosos é bastante diferenciado dos jovens e adultos, exigindo mais recursos, tanto pelo custo dos procedimentos,quanto pelo maior nível de utilização hospitalar desse grupo ${ }^{3}$. Os idosos atingem a ocupação de $20 \%$ dos leitos hospitalares e, em média, o tempo de internação é quase quatro vezes maior que a média para o total da população ${ }^{4}$.

As doenças respiratórias e circulatórias, entre os idosos, configuraram as principais causas de morbidade e o manejo adequado dessas doenças, em sua maioria crônicas, é negligenciado com frequência no cenário hospitalar ${ }^{5}$. O delineamento das especialidades médicas no cenário hospitalar dificulta a identificação de características e outros aspectos específicos do envelhecimento, ação esta que se torna relevante, uma vez que o contingente de idosos hospitalizados vem crescendo significativamente ${ }^{6}$.

A hospitalização por si só impõe um determinado grau de imobilidade e, para o paciente idoso, o declínio da capacidade funcional pode ser um pouco mais acelerado do que para as pessoas das demais faixas etárias. O comprometimento da capacidade funcional em idosos hospitalizados pode ser causado por inúmeros fatores tais como a idade avançada, a própria doença, os procedimentos médico-cirúrgicos, até a restrição no leito, os quais acarretam diminuição da sua mobilidade, maior risco de infecções hospitalares, uso de vários medicamentos, desnutrição e risco de quedas ${ }^{7}$.

O idoso passa por um evento estressante ao ser retirado de seu ambiente familiar, de sua rotina diária e ser inserido em um novo ambiente, com normas pré-estabelecidas e convivência com pessoas desconhecidas. A hospitalização torna-se então um evento complexo, peculiar e, potencialmente, perigoso para os idosos, sendo capaz de levar ao declínio ou perda da capacidade funcional ${ }^{8}$. O ambiente hospitalar impõe a pessoa novos enfrentamentos como uma série exames e procedimentos, que associados ao distanciamento da residência e dos familiares, medo do desconhecido e pensamentos relacionados à morte, aumentam a vulnerabilidade dos idosos 9 .

Um estudo realizado por Prado ${ }^{10}$, identificou que quanto maior a idade, maior é a presença dos sintomas depressivos. Ademais, Silva et al (2014) concluíram que os idosos que possuem dependência parcial ou total apresentaram mais suscetibilidade de desenvolver sintomas depressivos que os idosos independentes ${ }^{11}$. Nessa perspectiva, a implementação de ações de ordem preventiva encontra sua viabilidade através de promoção e estímulo de uma vida associativa e saudável, com a realização de atividades de recreação, atividades físicas e atividades culturais para os idosos ${ }^{12}$.

Considerando esses aspectos, em 2012 foi criado o Projeto de Lei $n^{\circ}$ 1788/2012, que cria o Programa "Amigo do Idoso" em todo o Estado do Rio de Janeiro. De acordo com o projeto, o Programa "Amigo do Idoso" deve ser implantado, nas clínicas de idosos, casas de repouso e nas enfermarias de longa permanência dos hospitais, públicos ou privados, em todo o Estado do Rio de Janeiro, contando com o apoio das Secretarias de Estado de Saúde e Assistência Social, que deverão coordenar o trabalho dos voluntários e dos profissionais na área da saúde e da assistência social, oferecer suporte material e didático. Além disso, tem como objetivo principal reunir voluntários para desenvolver atividades recreativas, sociais e culturais com os idosos internados, tendo como finalidade o estímulo ao raciocínio e a coordenação motora ${ }^{13}$.

A justificativa para criação deste projeto de lei consiste na assertiva de que a depressão é maior mal que acomete a população de idosos, seja por internação prolongada, por solidão ou descaso e abandono dos familiares. Nesse sentido, o projeto considera que faz-se necessário um programa que preencha esse vazio, levando atividades diversas como terapia ocupacional, lazer e socialização, que estimulem o raciocínio e a coordenação motora. Considera também que a grande maioria dos idosos, já sofrem com doenças crônicas, muitas vezes com limitações físicas, que dificultam a sua locomoção, consequentemente o acesso ao lazer $^{13}$.

Com base no Projeto de Lei supracitado e nas novas demandas impostas pelo envelhecimento populacional, principalmente no setor da saúde e no que se refere à hospitalização de idosos, um Hospital Estadual da Baixada Litorânea, no estado do Rio de Janeiro, foi o primeiro da região a contar com um projeto direcionado para os pacientes acima de 60 anos, tornando-se um Hospital Amigo do Idoso.

Nesse sentido, este estudo tem como objetivo relatar o processo de implantação do programa Hospital Amigo do Idoso, em um hospital da Baixada Litorânea, no estado do Rio de Janeiro, descrevendo as mudanças ambientais e assistenciais ocorridas durante sua implantação.

\section{MÉTODO}

Trata-se de um estudo descritivo, apresentado na forma de relato de experiência. $O$ hospital onde a experiência foi 
realizada é uma unidade de média e alta complexidade, com emergência regulada, que atende toda a população referenciada da Região da Baixada Litorânea do Rio de Janeiro e possui um grande índice de internações de pacientes idosos. Os atendimentos ocorrem por meio das seguintes unidades especializadas: trauma, terapia intensiva adulta, pediátrica e neonatal, emergências neurocirúrgicas, ortopedia, medicina interna, cirurgia geral e clínica de diálise, sendo esta última terceirizada.

Devido ao grande número de hospitalização de idosos, observou-se a necessidade de implantação de mudanças ambientais e assistenciais, no intuito de compatibilizar as ações de cuidado multiprofissionais e o ambiente às reais demandas do envelhecimento.

Sendo assim, o referido hospital foi o primeiro da região da Baixada Litorânea do estado do Rio de Janeiro a implantar o programa Hospital Amigo do Idoso, em novembro de 2015, com uma proposta de atendimento diferenciado, assistência de qualidade e prevenção de processos incapacitantes, por meio de ações de manutenção e recuperação da capacidade funcional, autonomia e independência. Atenção especial foi dedicada à prevenção de complicações inerentes à internação prolongada, como as consequências da inatividade no leito, inapetência, os efeitos colaterais das medicações, ambiente inadequado para o repouso dos pacientes, prevenção de quedas e de declínio da capacidade funcional.

\section{RESULTADOS E DISCUSSÃO}

Inicialmente, toda a equipe multiprofissonal de saúde recebeu capacitação, para prestar assistência adequada aos idosos, por meio de curso oferecido pela própria instituição, ministrado pelo diretor proponente e responsável pela implantação do programa. As demais equipes, profissionais administrativos, equipe de limpeza, equipe de recepção, e todos os demais profissonais que atuavam no âmbitodo hospital, também receberam preparo para lidar com a clientela idosa hospitalizada, através de comunicado interno e treinamento com os respectivos coordenadores de equipe. Toda a mobilização da comunidade hospitalar, para a implantação do programa, gerou a sensibilização de todos os envolvidos para as questões relativas à saúde do idoso hospitalizado.

A sensibilização dos profissionais de saúde que trabalham com idosos é uma das estratégias para qualificar o cuidado à saúde do idoso. Essa estratégia contribui para o engajamento profissional na identificação precoce de fatores de risco para fragilização, assim como na implementação de medidas preventivas para os eventos adversos à saúde do idoso ${ }^{14}$.

$\mathrm{Na}$ continuidade da proposta, com a intenção de proporcionar um ambiente mais amistoso e apropriado para o idoso, foram realizadas mudanças e adaptações na organização física das enfermarias, sendo elas: Dois leitos preferenciais por enfermarias, próximos às janelas, para estimular a noção temporal, pegar o sol da manhã e proporcionar uma distração com a vista do ambiente externo; Redução do ruído e luminosidade a partir das 22 horas; Sanitários mais seguros, por meio da instalaçãode barras de apoio, prevenindo as quedas.

Idosos institucionalizados, tanto em hospitais quanto em instituições de longa permanência, geralmente caem com maior frequência, evidenciando a necessidade de implantação de medidas preventivas relacionadas às quedas nesses locais. Nos hospitais, as quedas em pessoas idosas estão relacionadas a fatores intrínsecos aos pacientes, a saber: idade avançada, déficit de equilíbrio e marcha, incontinência urinária, comprometimento cognitivo, baixa escolaridade, presença de disfunção visual e outros. Os fatores extrínsecos estão relacionados a algumas condições do ambiente hospitalar e referentes a situações assistenciais, como uso de medicamentos hipnóticos, ansiolíticos e laxativos, entre outros ${ }^{15}$.

Além das ações de ordem física nas enfermarias, outras se destacaram: Identificação da equipe multiprofissional em um painel específico, através das cores dos uniformes e nomes, facilitando a compreensão dos idosos e acompanhantes, quanto a função de cada profissional dos plantões noturnos e diurnos; Incentivo ao banho de aspersão; Avaliação de risco nutricional; Atividade física de baixa intensidade e atividade recreativa, de estímulo cognitivo e funcional, na área externa.

Foram realizadas mudanças no acolhimento dos pacientes idosos. $\mathrm{Na}$ internação, desde a chegada ao hospital até o momento da acomodação em seu devido leito, diversas medidas foram tomadas para facilitar o trâmite. Ao ser identificado como idoso, o prontuário do paciente recebeu o selo personalizado do programa e logo o leito foi disponibilizado.

Também foram estabelecidas mudanças assistenciais da equipe multiprofissional. A implantação do programa Hospital Amigo do Idoso ocorreu devido ao engajamento de todos os profissionais envolvidos, desde a admissão do paciente até a alta hospitalar. As condutas a serem tomadas para favorecer a melhora e a alta precoce dos idosos foram minuciosamente discutidas entre a equipe multiprofissional, no intuito de prevenir o desenvolvimento de fragilidades.

A fragilidade é considerada uma síndrome multidimensional, que envolve uma interação de fatores biológicos, psicológicos e sociais no curso de vida individual, que resulta em maior vulnerabilidade, associada a maiores riscos de desfechos clínicos adversos como delírio, declínio funcional, mobilidade prejudicada, quedas, retraimento social, aumento da morbimortalidade e hospitalização ${ }^{16}$.

Com relação às necessidades de assistência dos idosos internados, foram confeccionados dois impressos para avaliação das condições de saúde e necessidades assistenciais dos idosos. 0 primeiro consistiu em perguntas sobre as demandas de cuidado, em forma de check-list, que foram respondidas marcando-se sim ou não, por meio de entrevista e exame físico detalhado, e também continha as 
possíveis intervenções para cada demanda apresentada. 0 segundo impresso consistiu em fatores de risco para a saúde dos idosos e intervenções cabíveis a serem implementadas pela equipe multiprofissional, para minimizar os riscos e comprometimentos.

Após a internação dos idosos, foram feitas as considerações sobre as necessidades e prioridades de assistência, através dos referidos impressos, que foram utilizados no momento da entrevista e associados ao exame físico detalhado, realizado primeiramente por um médico exclusivo do programa. Depois, as intervenções necessárias foram discutidas e implementadas por toda a equipe multiprofissional. 0 quadro 1 mostra uma síntese dos principais aspectos prioritários de saúde do idoso, considerando-se fatores de risco, e as intervenções a serem implementadas pela equipe.

A identificação de sinais e sintomas preditores da fragilização nos idosos, principalmente os hospitalizados, auxilia no desenvolvimento de intervenções e estratégias para minimizar efeitos desfavoráveis à saúde. 0 cuidado aos idosos fragilizados, bem como aos que apresentem riscos para a fragilidade, pode ser potencializado com o envolvimento de uma equipe interdisciplinar, que deve estar preparada para identificar e intervir nos problemas de saúde, visando a independência, autonomia, funcionalidade e qualidade de vida dos idosos ${ }^{14}$.

É fundamental que os profissionais da saúde tenham conhecimento dos aspectos específicos dos idosos e do processo de envelhecimento, para que os sinais e sintomas do surgimento de comprometimentossejam previamente diagnosticados, principalmente durante a hospitalização, a fim de evitar o agravamento, proporcionando planos terapêuticos que garantam sua execução em curto, médio e longo prazo, desde a promoção até a reabilitação das funções ${ }^{6}$.

Com o intuito de esclarecer os objetivos do programa, as mudanças adotadas e sua relevancia para a saúde dos pacientes idosos, elaborou-se um folder informativo que foi distribuído para os pacientes e familares, facilitando a compreensão e o envolvimento dessas pessoas.

Após uma avaliação preliminar do programa, através de dados contidos nos livros de registros de internação, foi possível verificar que o tempo de internação dos pacientes idosos diminuiu, em relação aos meses correspondentes de anos anteriores. Esse resultado se deve ao planejamento de alta precoce, que objetivou a prevenção do desenvolvimento de incapacidades e diminuição dos custos hospitalares.

No ato da alta hospitalar, os familiares foram orientados quanto a continuidade dos cuidados no ambiente domiciliar, os idosos receberam alta com avaliação da nutrição e dieta específica para sua patologia, agendamento de curativos com a Comissão de Curativos, além de encaminhamentos para outras especialidades, tais como oftalmologista e cardiologia, conforme necessidades identificadas durante a internação.
Quadro 1. Aspectos prioritários de saúde do idoso e intervenções

\begin{tabular}{|c|c|}
\hline $\begin{array}{l}\text { ASPECTOS } \\
\text { PRIORITÁRIOS }\end{array}$ & INTERVENÇÕES \\
\hline $\begin{array}{l}\text { Privação } \\
\text { sensorial }\end{array}$ & $\begin{array}{l}\text { Uso de equipamentos auxiliares: óculos e } \\
\text { aparelho auditivo. }\end{array}$ \\
\hline Desorientação & $\begin{array}{l}\text { Manter relógios visíveis no ambiente; } \\
\text { Manter calendário visível; Identificar o } \\
\text { nome da equipe de cuidados; Manter leito } \\
\text { próximo a janela. }\end{array}$ \\
\hline $\begin{array}{l}\text { Pertubação do } \\
\text { sono }\end{array}$ & $\begin{array}{l}\text { Reduzir o ruído e a luminosidade após } 22 \\
\text { horas (Desligar aparelhos de televisão; } \\
\text { Celulares no modo silencioso; Evitar } \\
\text { conversas desnecessárias); Ajustar horários } \\
\text { de cuidados, de modo a não prejudicar o } \\
\text { sono (Evitar medicação após } 22 \text { horas); } \\
\text { Ofertar chá morno; Evitar estímulo } \\
\text { farmacolígico do sono. }\end{array}$ \\
\hline Polifarmacologia & $\begin{array}{l}\text { Retirar da prescrição medicamentos } \\
\text { desnecessários; Cautela com a prescrição de } \\
\text { psicotrópicos. }\end{array}$ \\
\hline $\begin{array}{l}\text { Exames, } \\
\text { procedimentos } \\
\text { e dispositivos } \\
\text { desnecessários }\end{array}$ & $\begin{array}{l}\text { Avaliar a necessidade de exames de rotina, } \\
\text { muitas vezes desnecessários; Retirar, se } \\
\text { possível, acessos venosos, cateteres vesicais. }\end{array}$ \\
\hline Queixas álgicas & $\begin{array}{l}\text { Avaliar e realizar analgesia prontamente; } \\
\text { Avaliar analgesia regular ou antes de } \\
\text { procedimentos dolorosos. }\end{array}$ \\
\hline Isolamento social & $\begin{array}{l}\text { Incentivar a visitação de familiares e } \\
\text { amigos; Prolongar horário de visita; Ofertar } \\
\text { e estimular a participação em atividades } \\
\text { recreativas, de estímulo cognitivo e } \\
\text { funcional; Estimular e auxiliar nas visitas ao } \\
\text { ambiente externo. }\end{array}$ \\
\hline $\begin{array}{l}\text { Mobilidade } \\
\text { reduzida }\end{array}$ & $\begin{array}{l}\text { Estimular mobilidade e deambulação; } \\
\text { Sentar paciente fora do leito;Realizar } \\
\text { fisioterapia motora; Encaminhar ou } \\
\text { auxilar no banho de aspersão; Evitar o uso } \\
\text { desnecessário de fraldas geriátricas. }\end{array}$ \\
\hline $\begin{array}{l}\text { Desnutrição e } \\
\text { desidratação }\end{array}$ & $\begin{array}{l}\text { Evitar dietas zero, brandas e líquidas sem } \\
\text { necessidade; Oferta hídrica adequada; } \\
\text { Assegurar o uso de próteses dentárias; } \\
\text { Observar e avaliar motivos de recusa para } \\
\text { a dieta. }\end{array}$ \\
\hline \multicolumn{2}{|c|}{$\begin{array}{l}\checkmark \text { Reavaliar e discutir os aspectos frequentemente, } \\
\quad \text { readequando às demandas apresentadas; } \\
\checkmark \text { Prevenir quedas; } \\
\checkmark \text { Estabelecer um limite para continuação ou } \\
\text { descontinuação das medidas terapêuticas; } \\
\checkmark \text { Avaliar possibilidade de alta hospitalar precoce; } \\
\checkmark \text { Orientar idosos e familiares quanto a continuidade dos } \\
\text { cuidados no domicílio; } \\
\checkmark \text { Encaminhar para serviços especializados necessários. }\end{array}$} \\
\hline
\end{tabular}

Fonte: elaborado pelos autores.

\section{CONCLUSÃO}

A implantação do programa Hospital Amigo do Idoso significou uma importante estratégia de adequação ambiental e das práticas assistenciais às necessidades dos idosos hospitalizados, com foco nas demandas inerentes ao envelhecimento, muitas vezes acentuadas pelos processos de adoecimento e hospitalização. 
Essa iniciativa, liderada pela direção do hospital, representou um esforço institucional que reuniu diferentes profissionais. Sua implantação possibilitou mudanças fundamentais na instituição, como adequações físicas nas enfermarias, leitos prioritários para idosos, assistência baseada em decisões multiprofissionais, entre outras conquistas. A redução do tempo de internação dos idosos contribui para o não desenvolvimento de incapacidades relacionadas a internação prolongada.

Apesar dessas conquistas ainda é necessário um aperfeiçoamento e fortalecimento do programa, além de maior divulgação a fim de que esta iniciativa sirva de incentivo e seja tomada por outras unidades de saúde que prestam assistência às pessoas idosas.

A dificuldade encontrada na implantação do programa refere-se a ausência de um espaço ou enfermaria que pudesse ser exclusiva para assistência aos pacientes idosos, o que permitiria melhores adequações. Em contrapartida, como fator facilitador, detacou-se o engajamento de todos os profissionais no processo de implantação do programa, dos administrativos até os assistenciais, facilitando todo o processo desde a baixa até a alta hospitalar.

A experiência aqui relatada representa um registro do engajamento institucional e multiprofissional, centrado nos pacientes idosos hospitalizados. Esta é uma iniciativa viável, para garantir maior resolutividade das ações voltadas para o idoso no ambiente hospitalar e para impedir que hospitalização possa ser considerada um risco para esse paciente.

Cabe desejar que este relato contribua para fomentar iniciativas de adequação de outras unidades de saúde às demandas específicas do envelhecimento, aliadas ao processo de adoecimento do idoso que necessita ser hospitalizado e assistido em um ambiente seguro, com uma equipe multiprofissional preparada para saber avaliar e planejar uma assistência efetiva e acolhedora a pessoa idosa.

\section{REFERÊNCIAS}

1. IBGE-Instituto Brasileiro de Geografia e Estatística (Brasil). Ministério do Planejamento, Orçamento e Gestão. Diretoria de Pesquisas. Coordenação de População e Indicadores Sociais. Estudos e Pesquisas. Informação Demográfica e Socioeconômica - Indicadores Sociodemográficos e de Saúde no Brasil. Rio de Janeiro [Internet]. 2009 [Acesso em 2016 jan 17]. Disponível em: http://www.ibge.gov.br/home/estatistica/ populacao/indic_sociosaude/2009/indicsaude.pdf.

2. Brasil. Lei $n^{\circ} 10.741$, de $1^{\circ}$ de outubro de 2003. Dispõe sobre o Estatuto do Idoso e dá outras providências. Brasília-DF [Internet]. 2003 [Acesso em 2016 jan 19]. Disponível em: http://www.planalto.gov.br/CCivil_03/leis/2003/L10.741.htm

3. Borges GM, Campos MB, Silva LGC. Transição da estrutura etária no Brasil: oportunidades e desafios para a sociedade nas próximas décadas. In: Ervatti LR, Borges GM, Jardim AP, organizadores. IBGE. Mudança Demográfica no Brasil no Início do Século XXI: Subsídios para as projeções da população; 2015.p. 138-51.
4. Santos M. Epidemiologia do envelhecimento. In: Nunes MI, Ferretti REL, Santos M. Enfermagem em geriatria e gerontologia. Rio de Janeiro: Guanabara Koogan; 2012. p. 4-8.

5. Rabelo LPO, Vieira MA, Caldeira AP, Costa SM. Perfil de idosos internados em um hospital universitário. REME-Rev.Min. Enferm [Internet]. 2010 set [Acesso em 2016 jan 17]; 14(3): 293-300. Disponível em: http://bases.bireme.br/cgibin/wxislind.exe/iah/ online/?IsisScript=iah/iah. $x$ is $\&$ src $=$ google $\&$ base $=$ LILACS $\&$ lan $g=p \&$ nextAction=lnk\&exprSearch=578177 \&indexSearch=ID.

6. Sousa RM, Santana RF, Espírito Santo FH, Almeida JG, Alves LAF. Diagnósticos de enfermagem identificados em idosos hospitalizados: associação com as síndromes Geriátricas. Esc. Anna Nery [Internet]. 2010 dez [Acesso em 2016 jan 17]; 14(4): 732-41. Disponível em: http://www.scielo.br/scielo. php?pid=S1414-81452010000400012\&script=sci_arttext.

7. Menezes C, Oliveira VRC, Menezes RL. Repercussões da hospitalização na capacidade funcional de idosos. RevMovimento [Internet]. 2010 [Acesso em 2016 jan 18]; 3(2):76-84. Disponível em: http://www.nee.ueg.br/seer/index. php/movimenta/article/viewFile/358/317.

8. Lourenço TM, Lenardt MH, Kletemberg DF, Seima MD, Carneiro NHK. Functional independence of long-living elderly at hospital admission.Textocontextoenferm [Internet]. 2014 set [Acesso em 2016 jan 18]; 23(3): 673-9. Disponível em: http:// dx.doi.org/10.1590/0104-07072014001500013.

9. Duarte FM, Wanderley KS. Religião e espiritualidade de idosos internados em uma enfermaria geriátrica. Psicologia: Teoria e Pesquisa. Brasília [Internet]. 2011 [Acesso em 2016 jan 18]; 27 (1):49-53. Disponível em: http://www.scielo.br/scielo. php?pid=S0102-37722011000100007 \&script=sci_arttext.

10. Prado CP. Perfil dos idosos internados na unidade de clínica médica de um hospital geral terciário. [Dissertação]. São Paulo: Universidade de São Paulo, Ribeirão Preto [Internet]. 2011 [Acesso em 2016 jan 18]. Disponível em: http://www. teses.usp.br/teses/disponiveis/22/22132/tde-27032012153744/pt-br.php.

11. Silva PCS, Fernandes ACBC, Terra FS. Avaliação da depressão e da capacidade funcional em idosos com doença de Parkinson. Rev. Enferm UFPE [Internet]. 2014 [Acesso em 2016 jan 19]; 8(7):1920-7 Disponível em: www.revista.ufpe. br/revistaenfermagem/index.php/revista/article/.../9499.

12. Reis LA, Sampaio LS, Costa AN, Mascarenhas CHM. Influência do quadro depressivo na capacidade funcional de idosos institucionalizados. C\&D-Revista Eletrônica da Fainor [Internet]. 2011 [Acesso em 2016 jan 19]; 4(1):62-72. Disponível em: http://srv02.fainor.com.br/revista/index.php/ memorias/article/view/103.

13. RiodeJaneiro.AssembleiaLegislativadoEstadodo RiodeJaneiro. Projeto de Lei $n^{\circ}$ 1788/2012. Cria o programa "Amigo do Idoso" em todo o estado do Rio de Janeiro [Internet]. 2012 [Acesso em 2016 jan 18]. Disponível em: http://alerjln1.alerj.rj.gov. br/scpro1115.nsf/1061f759d97a6b24832566ec0018d832/ e7199563c4b9ba5f83257a9b0052d75f?OpenDocument.

14 Remor CB, Bós AJG, Werlang MC. Características relacionadas ao perfil de fragilidade no idoso. Scientia Medica [Internet]. 2011 [Acesso em 2016 jan 22]; 21(3):107112. Disponível em: http://web.a.ebscohost.com/ abstract? direct=true \& profile=ehost $\&$ scope $=$ site $\&$ auth type $=$ crawler $\&$ jrnl $=18065562 \& A N=67494818 \& \mathrm{~h}=$ lee- 
56i8vcs\%2fvMimwjbTc3RtQrBHTyWvDMqGx9sUcySoVP6iCq\% 2fIMNYBTgcrSHk0cP\%2bUgsqZi\%2b0Z7BulEDh\%2b$4 \mathrm{wQ} \% 3 d \% 3 d \& c r l=c \&$ resultNs=AdminWebAuth \& resultLocal=ErrCrlNotAuth \&crlhashurl=login.aspx\%3fdirect\%3dtrue\%26profile\%3dehost\%26scope\%3dsite\%26authtype\%3dcrawler\%26jrnl\%3d18065562\%26AN\%3d67494818

15. Abreu HCA, Reiners AAO, Azevedo RCS, Silva AMC, Abreu DROM, Oliveira AD. Incidência e fatores preditores de quedas de idosos hospitalizados. Rev Saúde Pública [Internet]. 2015 [Acesso em 2016 jan 22]; 49(37):1-9. Disponível em: http://www.scielo.br/pdf/rsp/v49/pt_00348910-rsp-S0034-89102015049005549.pdf

16. Oliveira DR, Bettinelli LA, Pasqualotti A, Corso D, Brock F, Erdmann AL. Prevalência de síndrome da fragilidade em idosos de uma instituição hospitalar. Rev. Latino-Am. Enfermagem [Internet]. 2013 [Acesso em 2016 jan 23]; 21(4):1-8. Disponível em: http://www.scielo.br/pdf/rlae/ v21n4/pt_0104-1169-rlae-21-04-0891.pdf. 